\title{
Statistical modeling of the EUR/DZD returns with infinite variance distribution
}

\author{
Ouadjed Hakim \\ Mascara University, Algeria \\ Faculty of Economics, Business and Management \\ Mathematics Laboratory, Djillali Liabes University of Sidi Bel Abbes, Algeria \\ o_hakim77@yahoo.fr
}

\begin{abstract}
The stable laws, generalization of the Gaussian law, constitute a very rich class of probability distributions, very interesting for the modeling of numerous financial phenomena which present a great variability. In this paper, we are interested in estimating some extreme risk measures at a high probability $p$ (the VaR "value-at-risk" and the TCE "Tail Conditional Expectation") for the series of the EUR exchange rate against the DZD (Algerian dinar) using the lévy-stable distribution..
\end{abstract}

Keywords: Extreme value theory, Lévy-stable law, Tail index.

\section{Risk measures}

Risk is always a concern in our lives. We face different types of daily risk among them the financial risk that can be distinguished at market risk, activity risk and credit risk. However, it is not at all easy to give it a precise definition. In general, risk means the possibility of damage or loss related to a given action. In finance and insurance, we are interested in assessing these risks using risk measures.

Let a random variable $X$. The measure of risk is a function $\mathcal{M}(X): X \rightarrow[0, \infty)$.

According to Artzner et al. (1999) a risk measure $\mathcal{M}($.$) is said to be coherent when it$ satisfies the following four coherence properties:

-(P1)-Positive homogeneity: $\mathcal{M}(\zeta X)=\zeta \mathcal{M}(X), \zeta>0$.

-(P2)-Translation invariance: $\mathcal{M}(X+\delta)=\mathcal{M}(X)+\delta, \delta \in \mathbf{R}$.

-(P3)-Monotony: For any random loss variables $X, Y$, with $X \leq Y$ in probability $\mathcal{M}(X) \leq \mathcal{M}(Y)$.

-(P4)-Sub-additivity : For any random variables $X, Y: \mathcal{M}(X+Y) \leq \mathcal{M}(X)+\mathcal{M}(Y)$.

Property (P1) states that the risk of a financial position is proportional to the size of the position. Property (P2) means that a sure loss of amount $\delta$ simply increases the risk by $\delta$. Property (P3) is a minimum requirement for a reasonable risk measure. What is controversial lies in the subadditivity requirement in property (P4), which basically means that "a merger does not create extra risk". 
The "VaR" (Value-at-Risk) risk measure associated to a random variable $X$, with the distribution function $F$, is defined by

$$
\operatorname{VaR}_{p}(X)=F_{X}^{-1}(p)=\inf \left\{x \in \mathbb{R} \mid F_{X}(x) \geq p\right\}, 0<p<1_{p}
$$

is one of the most widely used measures for the assessment of economic capital. In addition to its current use in market risk, it is often used as a benchmark for other types of risk and at the aggregate level of a company. Nevertheless, since the publication of the article by Artzner et al. (1999), the robustness of this measure is called into question by the fact that it does not satisfy the property of subadditivity ( $\mathrm{P} 4)$ and that can constitute a handicap for the VaR to measure the diversification of the risks. Artzner et al. (1999) proposed a risk measure called "Tail Conditional Expectation (TCE)" which is coherent and measures loss beyond VaR. This measure of risk is defined by

if $F$ is continuous then we have

$$
\operatorname{TCE} E_{p}(X)=\mathbf{E}\left[X \mid X>\operatorname{VaR}_{p}(X)\right] .
$$

$$
T C E_{p}(X)=\frac{1}{1-p} \int_{p}^{1} \operatorname{VaR}_{t}(X) d t
$$

From Albrecher et al. (2017) the relationship between $\operatorname{TCE}(X)$ and $\operatorname{VaR}_{p}(X)$ is given by

$$
\begin{aligned}
\operatorname{TCE}_{p}(X) & =\operatorname{VaR}_{p}(X)+E\left(X-\operatorname{VaR}_{p}(X) \mid X>\operatorname{VaR}_{p}(X)\right) \\
& =\operatorname{VaR}_{p}(X)+e\left(\operatorname{VaR}_{p}(X)\right),
\end{aligned}
$$

where $e$ is the mean excess function given by

$$
e(x):=E(X-x \mid X>x)=\frac{1}{S(x)} \int_{x}^{\infty} S(t) d t, x>0,
$$

with $S(x)=1-F_{X}(x)$ is the survival function.

Let the order statistic $X_{1, n} \leq X_{2, n} \leq \cdots \leq X_{n, n}$ associated with the sample $\left(X_{1}, X_{2}, \ldots, X_{n}\right)$ of $X$. The empirical estimate of $\operatorname{VaR}_{p}(X)$ is

$$
\widehat{\operatorname{VaR}}_{p}^{-m p}(X)=X_{[n p], n}
$$

and the empirical estimate of $T C E_{p}(X)$ is

$$
\widehat{T C E_{p}^{-m p}}(X)=\frac{1}{1-p}\left(\frac{1}{n} \sum_{k=[n p]+1}^{n} X_{k, n}+\left(\frac{[n p]}{n}-p\right) X_{[n p], n}\right)
$$

(see Rachev et al. (2008)), where $[x]$ is the integer part of $x$. 


\section{Lévy-stable distribution}

In the sixties, Mandelbrot's work on stock market fluctuations showed that the Gaussian model was not suitable for describing asset returns and the exchange rate. Mandelbrot (1997) then Fama (1965) proposed the Lévy-stable distribution, whose properties are very similar to those of heavy-tailed empirical distributions, as an alternative for modeling financial series.

A r.v. $X$ has a stable distribution if and only if for every integer $n \geq 1$ and for every i.i.d. family $X_{1}, X_{2}, \ldots, X_{n}$ of the same law as $X$, there exist two real numbers $\lambda_{n}>0$ and $\rho_{n}$ such that

$$
\frac{\left(X_{1}+X_{2}+\cdots+X_{n}\right)-\rho_{n}}{\lambda_{n}} \stackrel{D}{=} X_{y}
$$

where $\stackrel{D}{=}$ denotes equality in distribution. It is shown in Samorodnitsky and Taqqu (1994) that there exists a real constant $0<\alpha \leq 2$ such that $\lambda_{n}=n^{1 / \alpha}$.

Due to the importance of the class of stable distributions, it is necessary to describe them analytically. Their main disadvantage is that they do not have explicit forms for densities and distribution functions. The most common way of describing them is through their characteristic functions. For a complete description, one can refer to the book of Samorodnitsky and Taqqu (1994).

The characteristic function of a stable random variable is defined, for $t \in \mathbf{R}$, by

$$
\varphi_{X}(t)=\exp \left\{i \mu t-\sigma^{\alpha}|t|^{\alpha}\left(1+i \beta \frac{t}{|t|} w(t, \alpha)\right)\right\},
$$

where : $\alpha, \beta, \mu$ and $\sigma$ are constants such that: $-1 \leq \beta \leq 1,0<\alpha \leq 2, \sigma>0, \mu \in \mathbf{R}$ and

$$
w(t, \alpha)=\left\{\begin{array}{lll}
\operatorname{tg}\left(\frac{\alpha \pi}{2}\right) & \text { if } & \alpha \neq 1 \\
\frac{2}{\pi} \ln |t| & \text { if } & \alpha=1
\end{array}\right.
$$

We denote the Lévy-stable distribution by $S(\alpha, \mu, \beta, \sigma)$ where $\alpha$ is the stability index or the characteristic exponent, $\mu$ is the location or position parameter, $\beta$ is the asymmetry parameter and $\sigma$ is the scale parameter.

The most important properties of the stable random variables are the following:

1. If $X_{1} \sim S\left(\alpha, \mu_{1}, \beta_{1}, \sigma_{1}\right)$ and $X_{2} \sim S\left(\alpha, \mu_{2}, \beta_{2}, \sigma_{2}\right)$, independent, then :

$$
X_{1}+X_{2} \sim S\left(\alpha, \mu_{1}+\mu_{2}, \frac{\beta_{1} \sigma_{1}^{\alpha}+\beta_{2} \sigma_{2}^{\alpha}}{\sigma_{1}^{\alpha}+\sigma_{2}^{\alpha}},\left(\sigma_{1}^{\alpha}+\sigma_{2}^{\alpha}\right)^{1 / \alpha}\right) \text {. }
$$

2. If $X \sim S(\alpha, \mu, \beta, \sigma)$ and $a \in \mathbf{R}$, then $X+\alpha \sim S(\alpha, \mu+\alpha, \beta, \sigma)$.

3. If $X \sim S(\alpha, \mu, \beta, \sigma)$ and $a \in \mathbf{R}$, then

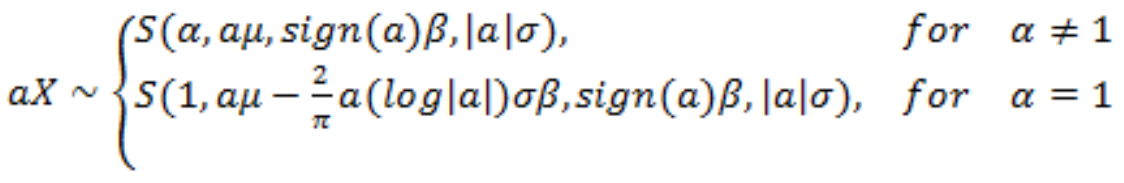


4. The $\alpha$-stables distributions class has a heavy tail properties. Let $X \sim S(\alpha, \mu, \beta, \sigma)$, then as $x \rightarrow \infty$, we have

where $C_{\alpha}=\frac{2}{\pi} \Gamma(\alpha) \sin \frac{\pi \alpha}{2}$.

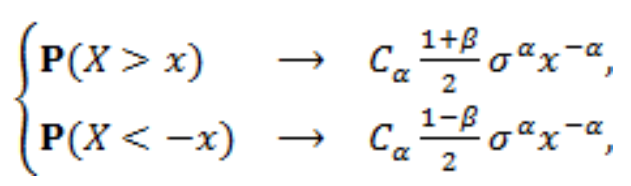

5. Let $X \sim S(\alpha, \mu, \beta, \sigma)$ with $\alpha \in] 0,2[$ then

$$
\left\{\begin{array}{lll}
E|X|^{m}<\infty, & \text { if } & m \in] 0, \alpha[ \\
E|X|^{m}=\infty, & \text { if } & m \in[\alpha, \infty[
\end{array}\right.
$$

Table 1: Moments of $\alpha$-stable random variable.

\begin{tabular}{|c|c|c|}
\hline & $0<\alpha \leq 1$ & $1<\alpha<2$ \\
\hline$E(X)$ & $\infty$ & $\mu$ \\
\hline$V(X)$ & $\infty$ & $\infty$ \\
\hline
\end{tabular}

The $\alpha$-stable distribution is characterized by four parameters from which one can estimate them, but the real disadvantage in this estimation is the absence of an explicit form of the density function. However, many numerical procedures have been proposed by several approaches. We can cite the empirical quantiles approach (see McCulloch (1986)), the empirical characteristic function approach (see Koutrouvelis (1980)) and the maximum likelihood (see Nolan (2001)).

For $\alpha$-stable law, there is an integral representation of the TCE obtained by Stoyanov et al. (2006). In particular, if $Z \sim S(\alpha, 0, \beta, 1), \alpha \neq 1$, we have

where

$$
T C E_{p}^{S}(Z)=\frac{\alpha}{(1-\alpha)} \frac{\left|V a R_{p}\right|}{\pi(1-p)} \int_{-\theta_{0}}^{\pi / 2} g(\theta) \exp \left(-\left|V a R_{p}\right|^{\alpha /(\alpha-1)} v(\theta)\right) d \theta
$$

and

$$
g(\theta)=\frac{\sin \left(\alpha\left(\theta_{0}+\theta\right)-2 \theta\right)}{\sin \left(\alpha\left(\theta_{0}+\theta\right)\right)}-\frac{\alpha \cos ^{2}(\theta)}{\sin ^{2}\left(\alpha\left(\theta_{0}+\theta\right)\right)}
$$

with

$$
v(\theta)=\left(\cos \left(\alpha \bar{\theta}_{0}\right)\right)^{1 /(\alpha-1)}\left[\frac{\cos (\theta)}{\sin \left(\alpha\left(\theta_{0}+\theta\right)\right)}\right]^{\alpha /(\alpha-1)} \frac{\cos \left(\alpha\left(\theta_{0}+\theta\right)-\theta\right)}{\cos (\theta)}
$$

$$
\bar{\theta}_{0}=\frac{1}{\alpha} \arctan \left(\bar{\beta} \tan \left(\frac{\pi \alpha}{2}\right)\right), \bar{\beta}=-\operatorname{sign}\left(\operatorname{VaR}_{p}\right) \beta .
$$

For $X \sim S(\alpha, \mu, \beta, \sigma)$, we have that $\sigma Z+\mu \sim X$, then

$$
T C E_{p}^{S}(X)=\sigma T C E_{p}^{S}(Z)+\mu .
$$

We can estimate $\alpha$ by the Hill estimator (1975) defined by

$$
\hat{\alpha}_{H}=\left(\frac{1}{k} \sum_{i=1}^{k} \log X_{n-i, n}-\log X_{n-k+1, n}\right)^{-1},
$$

where $X_{1, n} \leq X_{2, n} \leq \cdots \leq X_{n, n}$ are the order statistics and $k=k_{n}$ is an intermediate sequence such that 


$$
k \rightarrow \infty, k / n \rightarrow 0, n \rightarrow \infty \text {. }
$$

Weissman (1978) proposed the following semi-parametric estimator of a high quantile

$$
\widehat{\operatorname{VaR}}_{p}^{H}=X_{n-k_{2} n}\left(\frac{n(1-p)}{k}\right)^{-1 / \widehat{\alpha}_{H}} .
$$

It is known (see, e.g., Joe and $\mathrm{Li}(2011))$ that, for a $p \rightarrow 1$ and $\alpha>1$ we have

$$
T C E_{p}=\frac{\alpha}{\alpha-1} V a R_{p^{*}}
$$

Then we obtain the following estimator

$$
\widehat{T C} \bar{E}_{p}^{H}=\frac{\widehat{\alpha}_{H}}{\widehat{\alpha}_{H^{-1}}} X_{n-k, n}\left(\frac{n(1-p)}{k}\right)^{-1 / \widehat{\alpha}_{H}}
$$

\section{Application to real data}

If we have a financial series $\left(X_{i}\right), i=1, \ldots, n$, then the series of returns is defined as $R_{i}=\log \left(X_{i+1}\right)-\log \left(X_{i}\right), i=1, \ldots, n-1$.

Our study is carried out on the daily returns of the EURO exchange rates against the DZD (Algerian dinar) during the period from 30/09/2015 to 30/12/2016, the observations are shown in figure 1 . The data, taken from the website "http://www.finances.net/devisen/historique/euro-dinar_algérien-cours".

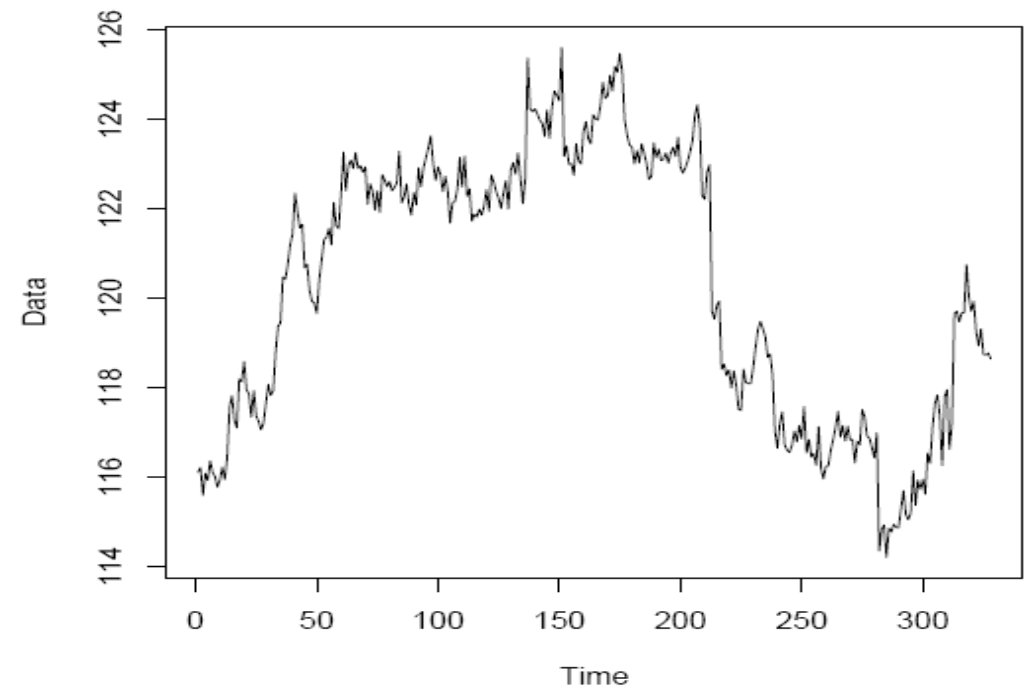

Figure 1: Exchange rate series EUR / DZD

The corresponding returns are shown in figure 2, which is characterized by a high variability. The main statistics concerning these returns are summarized in the table 2 and their distribution is plotted in figure 3. 
Table 2: Empirical characteristics of exchange rate returns EUR/DZD

\begin{tabular}{|c|c|}
\hline & $-6.606 \times 10^{-5}$ \\
\hline Mean & 0.004881274 \\
\hline Min & -0.02168 \\
\hline Max & 0.02702 \\
\hline
\end{tabular}

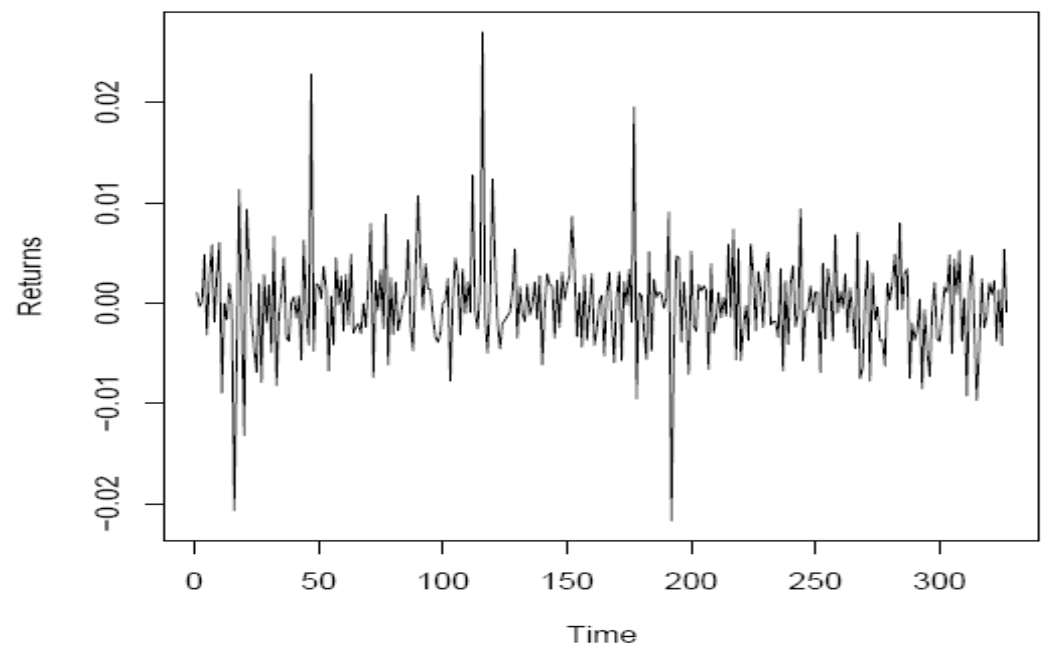

Figure 2: The daily returns series of EUR/DZD

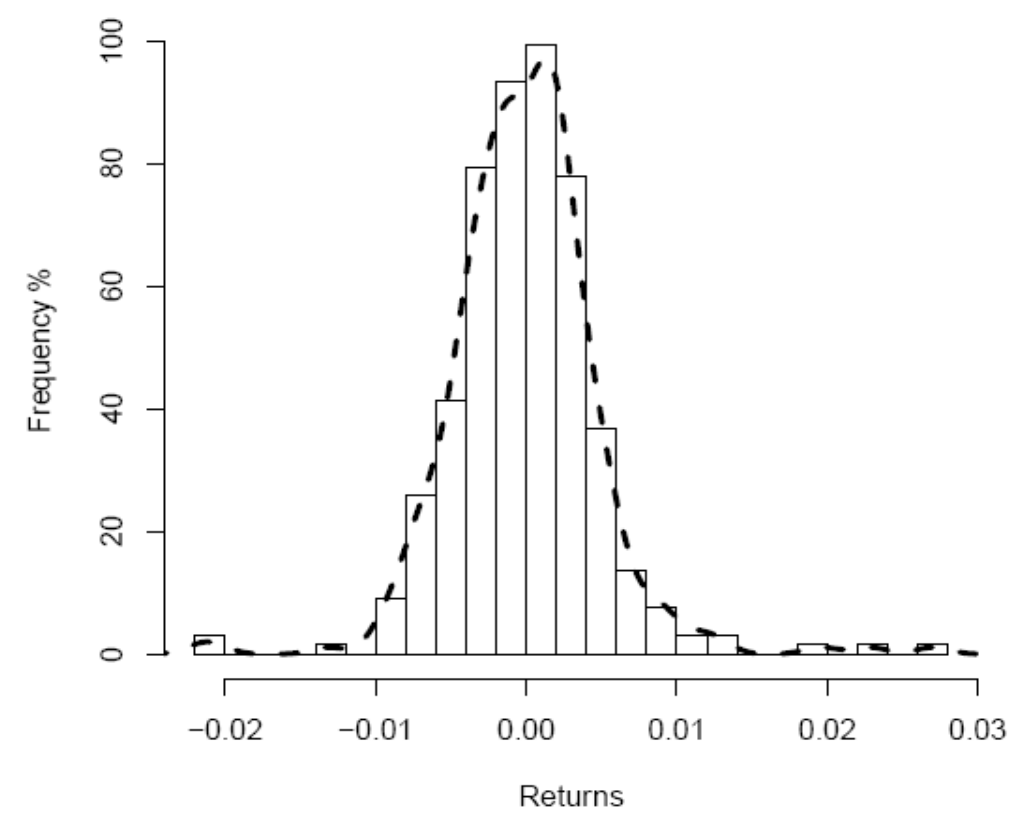

Figure 3: Distribution of the returns

The kurtosis value of this financial serie is $8.883>3$ which shows that the empirical law is leptokurtic. The qq-plot of figure 4 shows that the empirical law of returns is asymmetric and the tails of this law are thicker than those of the normal law. The fluctuations of the empirical variance in the figure 5 as a function of the increasing size of the sample shows the presence of jumps, which is a sign of non-convergence for the 
variance. On the other hand, the value of the $p$-value of the Shapiro-Wilk test is $9.965 \times 10^{-12}$. This also leads us to disregard the normality of the EUR/DZD returns.

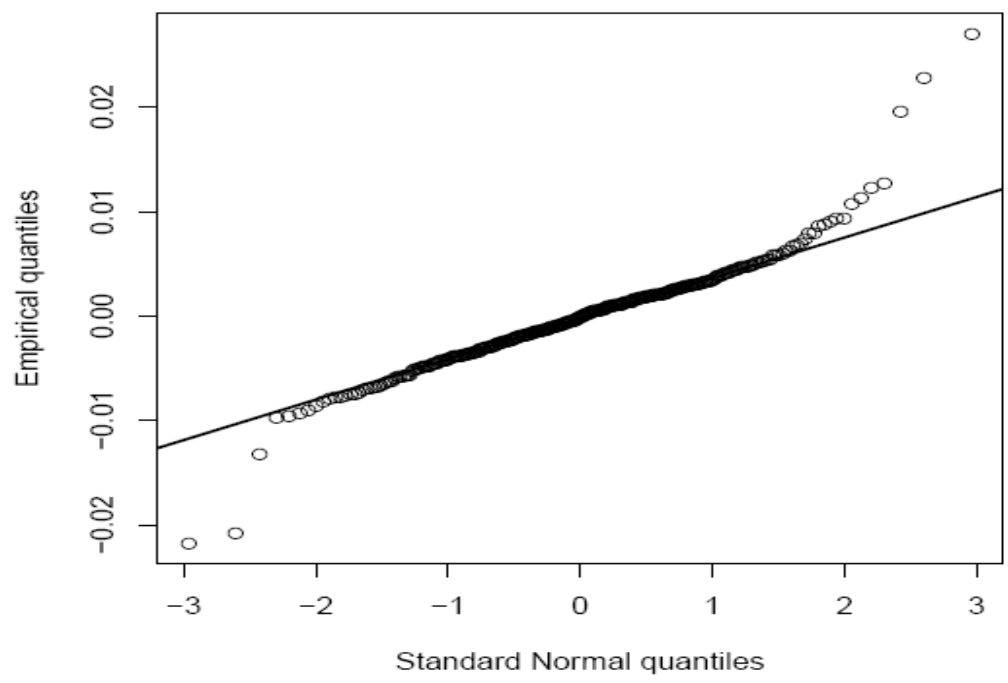

Figure 4: qq plot of EUR/DZD returns

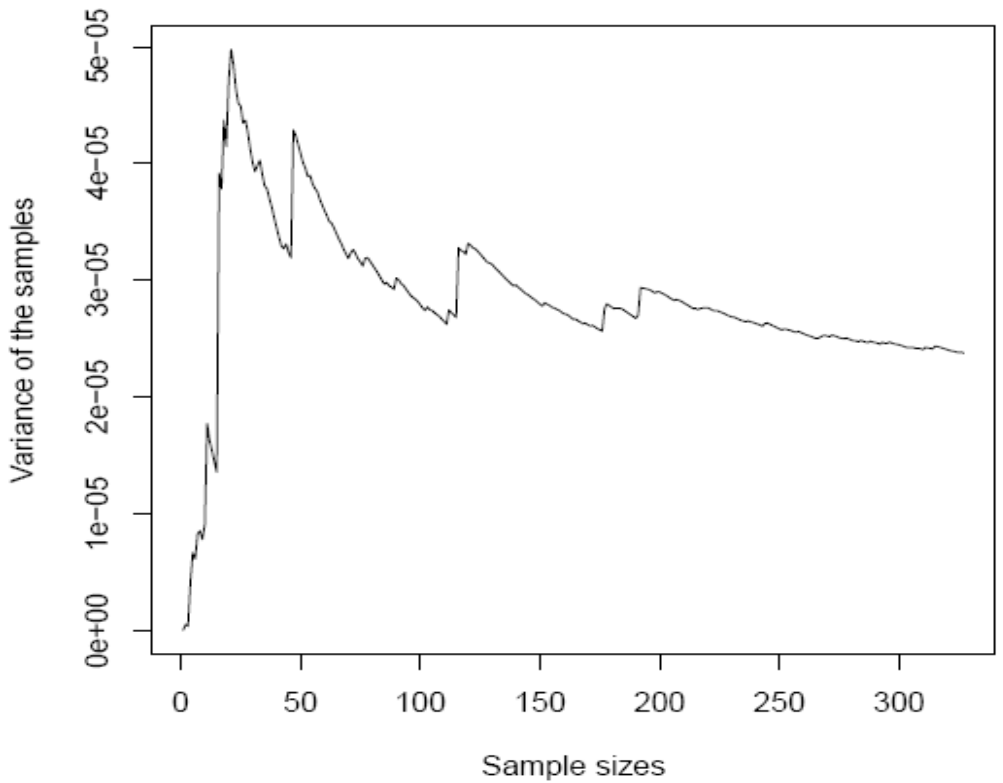

Figure 5: Fluctuations of empirical variance

The heavy yield tail, in combination with asymmetry, indicates that a stable distribution $S(\alpha, \mu, \beta, \sigma)$ is a suitable model for these returns, to estimate the parameters $\alpha, \mu, \beta$ and $\sigma$ we use the Koutrouvelis (1980) method.

The results obtained are summarized in the table 3.

Table 3: Stable parameters estimation of the EUR/DZD returns.

\begin{tabular}{|c|c|c|c|}
\hline$\hat{\alpha}$ & $\hat{\mu}$ & $\hat{\beta}$ & $\hat{\sigma}$ \\
\hline 1.772137 & -0.0001693724 & 0.1699817 & 0.002736298 \\
\hline
\end{tabular}


To see the quality of the data fit, the empirical density of the data is plotted in the figure 6 and the stable density $S(\hat{\alpha}, \hat{\mu}, \hat{\beta}, \hat{\sigma})$ which shows a good fit.

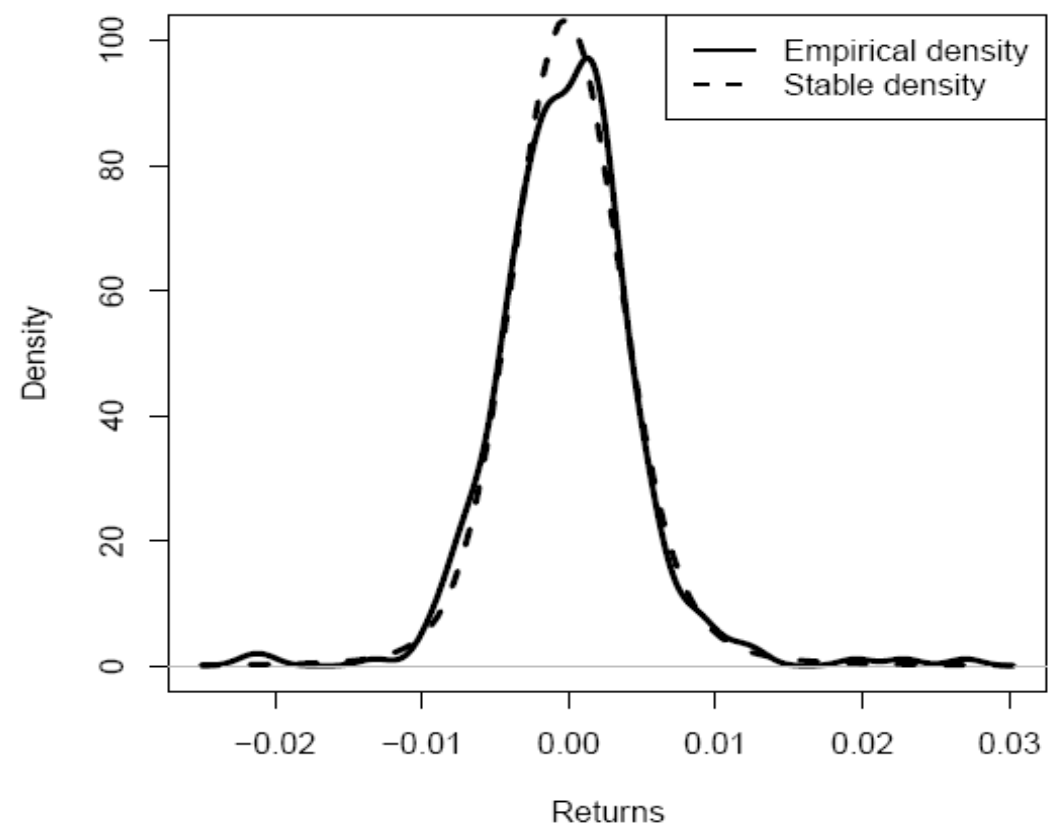

Figure 6: The quality of the adjustment.

In order to confirm this adjustment, we have the following table 4 which gives the $p$ value of Kolmogorov-Smirnov test and that of Anderson-Darling, we note that the stable law fit is better than that by the normal law at the significance level $5 \%$ especially in the tails.

Table 4: Adjustment test

\begin{tabular}{|c|c|c|}
\hline & \multicolumn{2}{|c|}{$\mathrm{p}$-value } \\
\hline Test & Kolmogorov-Smirnov & Anderson-Darling \\
\hline Stable adjustment & 0.9045 & 0.9435 \\
\hline Normal adjustment & 0.0281 & 0.02173 \\
\hline
\end{tabular}

For selecting an optimal $k$ of the Hill estimator in (5) we use an implementation of the procedure proposed in Guillou and Hall (2001) based on bias reduction. Then we find $k_{\text {opt }}=47$ and $\hat{\alpha}_{H}=1.939385$. To estimate the extreme quantiles $\left(\operatorname{VaR}_{0.95}^{S}, \operatorname{VaR}_{0.99}^{S}\right)$ of the $S(\hat{\alpha}, \hat{\mu}, \hat{\beta}, \hat{\sigma})$ and since the stable law does not admit an explicit form, they have been computed numerically using the qstable function of the fbasics package of the language R. To estimate the Tail Conditional Expectation $\left(T C E_{0.95}^{S}, T C E_{0.99}^{S}\right)$ we use the formulas (3) and (4). To estimate the extreme quantiles $\left(\operatorname{VaR}_{0.95}^{H}, V a R_{0.99}^{H}\right)$ we use the formula (6). To estimate the Tail Conditional Expectation $\left(T C E_{0.95}^{H}, T C E_{0.99}^{H}\right)$ we use the formula (8). In the table 5 we present the estimates of the risk measures VaR and TCE. For a comparison we also calculate $\widehat{V a R_{p}} \widehat{m p}^{-m p}$ and $\widehat{T C E_{p}^{m p}}$ using the formulas (1), (2) respectively. 
Table 5: Estimation of extreme quantiles and Tail Conditional Expectation of the returns.

\begin{tabular}{|c|c|c|}
\hline$p$ & 0.95 & 0.99 \\
\hline$\widehat{V a R_{p}^{-S}}$ & 0.007056682 & 0.013020659 \\
\hline$\widehat{V a R_{p}^{-H}}$ & 0.006770013 & 0.01552378 \\
\hline$\widehat{V a R_{p}^{-6 m p}}$ & 0.006758447 & 0.012663437 \\
\hline$\widehat{T C E_{p}^{-S}}$ & 0.01160754 & 0.02304002 \\
\hline$\widehat{T C E_{p}}{ }^{H}$ & 0.01397687 & 0.03204926 \\
\hline$\widehat{T C E_{p}}$ & 0.011903 & 0.02236173 \\
\hline
\end{tabular}

It can be seen that the estimated $\mathrm{VaR}$ and TCE with stable law is closer to the empirical $\mathrm{VaR}$ and TCE than estimated using the Hill estimator.

\section{Conclusion}

In this paper, we fit a Lévy stable model for the empirical distributions of EUR/DZD log returns, then we use some goodness-of-fit tests, in order to quantitatively the quality of the fit, we remark that the $\alpha$-stable distribution is better fitted to the returns than the Gaussian one. Using these heavy tailed distribution we evaluate the extreme risk considering two most often risk measures: the Value at Risk (VaR) and the Tail Conditional Expectation (TCE), we observe that the adjustment by a stable law gives better results than using the Hill estimator which have optimality properties only when the underlying distribution is close to Pareto.

There are a number of problems connected with VaR and TCE that need immediate attention. The assumption of independence of the return is always questionable. Some attempts have already been made in relaxing the assumption of independent and identical distribution for the returns. McNeil and Frey (2000) have considered the problem of estimation of TCE when the return process is ARCH and GARCH. Recently Ouadjed (2018) estimated VaR and TCE using the extremal index to model dependence, and the peaks-over-threshold (POT) method of extreme value theory (EVT) to model the tail behaviour of asset returns. However, further generalisation of $\mathrm{VaR}$ and TCE and its estimations for various models needs to be investigated.

\section{Acknowledgements}

The authors would like to thank the referee for careful reading and for their comments which greatly improved the paper. 


\section{References}

1. Albrecher, H., Teugels, J. L., \& Beirlant, J. (2017). Reinsurance: actuarial and statistical aspects. John Wiley \& Sons.

2. Artzner, P., Delbaen, F., Eber, J. M., \& Heath, D. (1999). Coherent measures of risk. Mathematical finance, 9(3), 203-228.

3. Fama, E. F. (1965). The behavior of stock-market prices. The journal of Business, 38(1), 34-105.

4. Guillou, A., \& Hall, P. (2001). A diagnostic for selecting the threshold in extreme value analysis. Journal of the Royal Statistical Society: Series B (Statistical Methodology), 63(2), 293-305.

5. Hakim, O. (2018). POT approach for estimation of extreme risk measures of EUR/USD returns. Statistics, Optimization \& Information Computing, 6(2), 240-247.

6. Hill, B. M. (1975). A simple general approach to inference about the tail of a distribution. The annals of statistics, 1163-1174.

7. Joe, H., \& Li, H. (2011). Tail risk of multivariate regular variation. Methodology and Computing in Applied Probability, 13(4), 671-693.

8. Koutrouvelis, I. A. (1980). Regression-type estimation of the parameters of stable laws. Journal of the American statistical association, 75(372), 918-928.

9. Mandelbrot, B. B. (1997). The variation of certain speculative prices. In Fractals and scaling in finance (pp. 371-418). Springer, New York, NY.

10. McCulloch, J. H. (1986). Simple consistent estimators of stable distribution parameters. Communications in Statistics-Simulation and Computation, 15(4), 11091136.

11. McNeil, A. J., \& Frey, R. (2000). Estimation of tail-related risk measures for heteroscedastic financial time series: an extreme value approach. Journal of empirical finance, 7(3-4), 271-300.

12. Nolan, J. P. (2001). Maximum likelihood estimation and diagnostics for stable distributions. In Lévy processes (pp. 379-400). Birkhäuser, Boston, MA.

13. Rachev, S., \& Stoyanov, S. W. (2008). Advanced Stochastic Models, Risk Assessment, and Portfolio Optimization, The Frank J. Fabozzi series.

14. Samorodnitsky, G., \& Taqqu, M. (1994). Non-Gaussian Stable Processes: Stochastic Models with Infinite Variance. Chapman ft Hall, London.

15. Stoyanov, S. V., Samorodnitsky, G., Rachev, S., \& Ortobelli Lozza, S. (2006). Computing the portfolio conditional value-at-risk in the alpha-stable case. Probability and Mathematical Statistics, 26(1), 1-22.

16. Weissman, I. (1978). Estimation of parameters and large quantiles based on the $\mathrm{k}$ largest observations. Journal of the American Statistical Association, 73(364), 812-815. 